\title{
Tidal deformations of neutron stars with elastic crusts
}

\author{
Fabian Gittins* and Nils Andersson \\ Mathematical Sciences and STAG Research Centre, \\ University of Southampton, Southampton SO17 1BJ, United Kingdom \\ Jonas P. Pereira \\ Nicolaus Copernicus Astronomical Centre, \\ Polish Academy of Sciences, Bartycka 18, 00-716, Warsaw, Poland
}

(Dated: May 11, 2020)

\begin{abstract}
With the first detections of binary neutron star mergers by gravitational-wave detectors, it proves timely to consider how the internal structure of neutron stars affects the way in which they can be asymmetrically deformed. Such deformations may leave measurable imprints on gravitational-wave signals and can be sourced through tidal interactions or the formation of mountains. We detail the formalism that describes fully-relativistic neutron star models with elastic crusts undergoing static perturbations. This formalism primes the problem for studies into a variety of mechanisms that can deform a neutron star. We present results for a barotropic equation of state and a realistic model for the elastic crust, which enables us to compute relevant quantities such as the tidal deformability parameter. We find that the inclusion of an elastic crust provides a very small correction to the tidal deformability. The results allow us to demonstrate when and where the crust starts to fail during a binary inspiral and we find that the majority of the crust will remain intact up until merger.
\end{abstract}

\footnotetext{
*f.w.r.gittins@soton.ac.uk
} 


\section{INTRODUCTION}

The confident detections of the binary neutron star merger events GW170817 and GW190425 through gravitational waves has heralded in an exciting new era for neutron star astrophysics [1, 2]. Among other things, neutron stars provide unique probes into the equation of state of matter at supranuclear densities, which remains a topical issue in astrophysics and nuclear physics. The equation of state encodes the microscopic nuclear interactions and plays a fundamental role in determining the configuration of neutron stars. At the macroscopic level, it manifests itself through observables such as the mass, radius and stellar moment of inertia (see, e.g., [3]). Terrestrial experiments are able to study and constrain the equation of state up to densities just below the nuclear saturation density $\rho_{\text {sat }}=2.7 \times 10^{14} \mathrm{~g} \mathrm{~cm}^{-3}$ (see, e.g., [4-7] for reviews on the subject) - markedly below the densities in neutron star cores. For this reason, we must look to the (neutron) stars for inspiration.

It has proven quite the endeavour to provide constraints on the internal structure of neutron stars through astrophysical observations; the observed range of masses is large, $M \sim 1.1-2.0 M_{\odot}[8]$, and, problematically, it would seem there is no current modelindependent technique to measure the radius. Various radius estimates have been made using X-ray spectroscopy from quiescent neutron stars [9], thermonuclear X-ray bursts [1012] and accretion-powered millisecond pulsars [13]. However, all these methods suffer from being susceptible to systematic errors $[14,15]$. Promisingly, the NICER mission is expected to be far less prone to such biases and their first results have provided relatively tight bounds on the radius of PSR J0030+0451 [16-19]. Different equation-of-state candidates predict for a neutron star of mass $M=1.4 M_{\odot}$ a radius in the range of $9 \mathrm{~km} \lesssim R \lesssim 14 \mathrm{~km}[6]$.

One of the exciting prospects of gravitational-wave observations is that they can provide model-independent constraints on the equation of state and, indeed, have done in the case of GW170817 [1, 20, 21]. The gravitational-wave signal emitted from inspiralling neutron stars differs slightly from that of inspiralling black holes. The very fact that neutron stars are extended bodies introduces finite-size corrections to the gravitational-wave signal. The dominant finite-size effect comes from the tidal deformation that each star's gravitational field induces on the other. Since this effect depends on the density distribution of the star, it may be used as a diagnostic to probe the neutron star interior. However, neutron stars 
are believed to have solid crusts close to their surfaces, which introduce further complexity into prospective descriptions of the interior [22]. There have been a number of studies of perturbations of neutron stars with elastic crusts. Most of these have assumed the Cowling approximation [23, 24] and only a few have accounted fully for relativistic effects [25, 26]. In recent years, there have been a couple of efforts in the direction of understanding the impact that the inclusion of an elastic crust makes on tidal deformations in neutron stars [27, 28].

In this work, we detail the formalism that describes static perturbations of non-rotating neutron stars with an elastic component. We do this because of slight inconsistencies found in the work of [27], also noted by [28] and [29]. We also find discrepancies in the analysis of [28]. The specific aim of this work is to quantify the role of an elastic crust in tidal deformations. The paper is organised as follows. To begin with, in Sec. II, we describe our approach to calculating static, even-parity linearised perturbations of a neutron star with fluid and elastic layers. In Sec III, we apply this formalism to calculate the tidal deformations of a neutron star with an elastic crust using a realistic equation of state and we discuss our results. We summarise and conclude in Sec IV.

We use the metric signature $(-,+,+,+)$ and work in geometric units with $G=c=$ 1. We adopt the usual Einstein summation convention where repeated indices indicate a summation. Early Latin characters $a, b, \ldots$ are used for spacetime indices and we reserve later characters $i, j, \ldots$ for spatial indices. We use primes to denote differentiation with respect to the radial coordinate.

\section{NEUTRON STAR PERTURBATIONS}

Since we are setting up the problem to study tidal deformations, it is appropriate to work in the adiabatic limit, where the variations in the tidal field are assumed to be slow compared to the timescale associated with the star's internal response [30]. For this reason, we focus on static perturbations of non-rotating neutron stars and we further assume that the unperturbed neutron star is relaxed - that is to say, the background is unstrained. This is the pertinent assumption for a widely-separated binary and it should be valid up to the point when the crust begins to fail due to built-up strain. We partition our neutron stars into three layers: an inner fluid core, an elastic crust and an outer fluid ocean. Therefore, the equilibrium configuration is straightforwardly described by the usual relativistic equations of 
stellar structure: the Tolman-Oppenheimer-Volkoff equations. The elastic crust will manifest itself at the linear perturbation level.

\section{A. The background configuration}

The interior of a static, spherically-symmetric star is described by the spacetime metric $g_{a b}$ given by the line element

$$
d s^{2}=g_{a b} d x^{a} d x^{b}=-e^{\nu} d t^{2}+e^{\lambda} d r^{2}+r^{2}\left(d \theta^{2}+\sin ^{2} \theta d \phi^{2}\right)
$$

where $\lambda(r)$ and $\nu(r)$ are metric functions of $r$. Since the star is static, the only non-vanishing component of the fluid four-velocity is the $t$ component. Thus, the four-velocity is

$$
u^{t}=e^{-\nu / 2}, \quad u^{i}=0 .
$$

The background configuration is a perfect fluid. The stress-energy tensor for a perfect fluid is

$$
T_{a b}=(\rho+p) u_{a} u_{b}+p g_{a b}=\rho u_{a} u_{b}+p \perp_{a b},
$$

where $\rho(r)$ is the energy density, $p(r)$ is the pressure and we have introduced the projection operator orthogonal to the fluid flow

$$
\perp_{a b} \equiv u_{a} u_{b}+g_{a b},
$$

which will be useful later.

One then solves the Einstein equations for this configuration and defines

$$
e^{\lambda} \equiv \frac{1}{1-2 m / r}
$$

where $m(r)$ is the gravitational mass enclosed in $r$. The mass is obtained from

$$
m^{\prime}=4 \pi r^{2} \rho
$$

The metric potential is described by

$$
\nu^{\prime}=\frac{2\left(m+4 \pi r^{3} p\right)}{r(r-2 m)}
$$

and by using the relativistic equation for hydrostatic equilibrium one finds

$$
p^{\prime}=-\frac{1}{2}(\rho+p) \nu^{\prime}=-\frac{(\rho+p)\left(m+4 \pi r^{3} p\right)}{r(r-2 m)} .
$$


Eqs. (6) are the Tolman-Oppenheimer-Volkoff equations. Provided an equation of state one can solve these differential equations through numerical integration to obtain a description of the neutron star background. It is useful to note that (6b) decouples from the other two equations. Therefore, one need only solve (6a) and (6c) to find the mass and radius of the star.

\section{B. Fluid perturbation equations}

The standard approach to computing stellar perturbations in general relativity is to follow [31]. We use the Regge-Wheeler gauge [32] and focus on static, even-parity $l \geq 2$ perturbations, which leads to the perturbed metric

$$
h_{a b}=\left(\begin{array}{cccc}
e^{\nu} H_{0} & H_{1} & 0 & 0 \\
H_{1} & e^{\lambda} H_{2} & 0 & 0 \\
0 & 0 & r^{2} K & 0 \\
0 & 0 & 0 & r^{2} \sin ^{2} \theta K
\end{array}\right) Y_{l m}
$$

where $H_{0}(r), H_{1}(r), H_{2}(r)$ and $K(r)$ describe the response of the spacetime to the perturbations and $Y_{l m}(\theta, \phi)$ is a spherical harmonic. The perturbed metric is sourced by the perturbations to the stress-energy tensor $\delta T_{a}{ }^{b}$. This coupling is described by the linearised Einstein equations,

$$
\delta G_{a}^{b}=8 \pi \delta T_{a}^{b}
$$

where $\delta G_{a}{ }^{b}$ is the perturbed Einstein tensor. The calculation of the perturbed Einstein tensor is rather laborious and not particularly insightful, so we simply state the result [33]:

$$
\begin{aligned}
2 \delta G_{a}{ }^{b}=\nabla^{c} \nabla_{a} h_{c}{ }^{b} & +\nabla_{c} \nabla^{b} h_{a}{ }^{c}-\nabla^{c} \nabla_{c} h_{a}{ }^{b}-\nabla^{b} \nabla_{a} h \\
& -2 R_{a}{ }^{c} h_{c}{ }^{b}-\left(\nabla^{d} \nabla_{c} h_{d}{ }^{c}-\nabla^{c} \nabla_{c} h-R_{c}{ }^{d} h_{d}{ }^{c}\right) \delta_{a}{ }^{b},
\end{aligned}
$$

where $h$ is the trace of the perturbed metric and $R_{a}^{b}$ is the Ricci tensor associated with the background spacetime.

To characterise the perturbations, we introduce the static displacement vector [27]

$$
\xi^{a}=\left[\begin{array}{c}
0 \\
r^{-1} W \\
r^{-2} V \partial_{\theta} \\
(r \sin \theta)^{-2} V \partial_{\phi}
\end{array}\right] Y_{l m}
$$


with functions $W(r)$ and $V(r)$ describing the radial and tangential displacements, respectively. In the context of relativistic perturbation theory the Lagrangian variation of the four-velocity is given as [34]

$$
\Delta u^{a}=\frac{1}{2} u^{a} u^{b} u^{c} \Delta g_{b c},
$$

where the Lagrangian perturbation of the metric is

$$
\Delta g_{a b}=h_{a b}+2 \nabla_{(a} \xi_{b)} .
$$

Here, the brackets around indices (...) denote symmetrisation. The Lagrangian perturbations are straightforwardly related to the Eulerian perturbations, denoted with $\delta$, by $\Delta=\delta+\mathcal{L}_{\xi}$, where $\mathcal{L}_{\xi}$ is the Lie derivative along $\xi^{a}$. Thus, the Eulerian perturbed four-velocity is

$$
\delta u^{a}=\perp^{a}{ }_{b} \mathcal{L}_{u} \xi^{b}+\frac{1}{2} u^{a} u^{b} u^{c} h_{b c},
$$

which has components

$$
\delta u^{t}=\frac{1}{2} e^{-\nu / 2} H_{0} Y_{l m}, \quad \delta u^{i}=0 .
$$

One should note that, given the displacement vector is static, the displacement vector components do not appear in the perturbed four-velocity.

A useful relation for later on comes from considering the Lagrangian change of the number density [34],

$$
\Delta n=-\frac{1}{2} n \perp^{a b} \Delta g_{a b},
$$

where $n$ is the background number density. Computing this explicitly gives

$$
\Delta n=-\frac{1}{2} n \perp_{\mathrm{g}} Y_{l m},
$$

where we have defined [27]

$$
\perp_{\mathrm{g}} \equiv \frac{2}{r^{2}}\left[r^{2}\left(K+\frac{1}{2} H_{2}\right)-l(l+1) V+r W^{\prime}+\left(1+\frac{1}{2} r \lambda^{\prime}\right) W\right] .
$$

The focus of this work will be on barotropic matter, where the energy density is a function of only the number density, $\rho=\rho(n)$, which means that

$$
\Delta \rho=\frac{d \rho}{d n} \Delta n=\mu \Delta n,
$$

where $\mu$ is the chemical potential. This also leads to the relations

$$
\Delta p=\frac{d p}{d \rho} \Delta \rho=c_{\mathrm{s}}^{2} \Delta \rho, \quad \delta p=c_{\mathrm{s}}^{2} \delta \rho,
$$


where we have identified the speed of sound, $c_{\mathrm{s}}$. We can use the Gibbs relation, $\rho+p=\mu n$, and combine (16) and (18) to show

$$
\Delta \rho=-\frac{1}{2}(\rho+p) \perp_{\mathrm{g}} Y_{l m}
$$

Therefore, by (19)

$$
\Delta p=-\frac{1}{2}(\rho+p) c_{\mathrm{s}}^{2} \perp_{\mathrm{g}} Y_{l m} .
$$

We also have, from the relation between Lagrangian and Eulerian variations,

$$
\Delta p=\delta p+\xi^{r} p^{\prime}=\delta p-\frac{1}{2 r}(\rho+p) \nu^{\prime} W Y_{l m}
$$

We will use (21) and (22) later on to close our system of equations for the crustal perturbations.

The matter content of the spacetime is encoded in the stress-energy tensor. To complete the specification for the linearised Einstein equations we use the stress-energy tensor for a perfect fluid (3) to obtain

$$
\delta T_{a}^{b}=(\delta \rho+\delta p) u_{a} u^{b}+\delta p \delta_{a}^{b}+(\rho+p)\left(\delta u_{a} u^{b}+u_{a} \delta u^{b}\right)
$$

where the perturbed quantities are to be expanded in spherical harmonics, e.g., $\delta \rho(r, \theta, \phi) \rightarrow$ $\delta \rho(r) Y_{l m}(\theta, \phi)$. Note that, with such an expansion, a summation over all $l, m$ is implied. However, for this analysis, it will be sufficient to calculate the perturbations for a given harmonic mode.

One obtains a system of coupled ordinary differential equations by inserting (9) and (23) into (8) which describe the perturbations in the fluid regions of the star. Conveniently, this system of equations simplifies to a single second-order differential equation [35]:

$$
\begin{aligned}
H_{0}^{\prime \prime}+\left(\frac{2}{r}+\frac{\nu^{\prime}-\lambda^{\prime}}{2}\right) H_{0}^{\prime} & +\left\{\frac{2}{r^{2}}-[2+l(l+1)] \frac{e^{\lambda}}{r^{2}}\right. \\
& \left.+\frac{9 \nu^{\prime}+5 \lambda^{\prime}}{2 r}-\nu^{\prime 2}+\frac{\nu^{\prime}+\lambda^{\prime}}{2 r c_{\mathrm{s}}^{2}}\right\} H_{0}=0 .
\end{aligned}
$$

For completeness, one can calculate the other metric perturbations from $H_{1}=0, H_{2}=H_{0}$ and

$$
[l(l+1)-2] e^{\lambda} K=r^{2} \nu^{\prime} H_{0}^{\prime}+\left[l(l+1) e^{\lambda}-2-r\left(\nu^{\prime}+\lambda^{\prime}\right)+r^{2} \nu^{\prime 2}\right] H_{0} .
$$

As we will see later, the equations which describe the perturbations in the elastic crust reduce to these fluid equations. At this point, it is worth remarking that, given the static 
nature of the problem, we are unable compute the displacement vector in the fluid, since the functions $W$ and $V$ do not appear in the perturbed stress-energy tensor. This issue was somewhat confused in the analysis of [28], who present equations for the components of the displacement vector. These quantities could be calculated by assuming the fluid regions of the star have a small, but non-zero, shear modulus. We do not do this in our analysis and treat those regions as perfect fluids, which seems more appropriate.

\section{Including elasticity}

As discussed previously, the background star is assumed to be in a relaxed state. This means that the contribution of the elastic crust only enters through the perturbed stressenergy tensor. For an elastic material with shear modulus $\check{\mu}$, the Lagrangian perturbation of the anisotropic stress tensor is [36]

$$
\Delta \pi_{a b}=-2 \check{\mu} \Delta s_{a b}
$$

where the perturbed strain tensor $\Delta s_{a b}$ is given by

$$
2 \Delta s_{a b}=\left(\perp^{c}{ }_{a} \perp^{d}{ }_{b}-\frac{1}{3} \perp_{a b} \perp^{c d}\right) \Delta g_{c d} .
$$

The anisotropic stress tensor is trace-free. Since the background is unstrained we simply find

$$
\delta \pi_{a}{ }^{b}=-\check{\mu}\left(\perp^{c}{ }_{a} \perp^{d}{ }_{b}-\frac{1}{3} \perp_{a b} \perp^{c d}\right) \Delta g_{c d} .
$$

We note that in the equivalent expression in [27] there is a difference of a factor of two. Summing the anisotropic stress tensor (27) and the fluid stress-energy tensor (3) and inserting these expressions into the perturbed Einstein equations (8) provides the information needed to describe perturbations in the crust.

Motivated by the analysis of [26], we define the following dimensionless variables which are related to the radial and perpendicular components of the traction:

$$
\begin{aligned}
T_{1} Y_{l m} & \equiv r^{2} \delta \pi_{r}^{r}=\frac{2 \check{\mu}}{3}\left[r^{2}\left(K-H_{2}\right)-l(l+1) V-2 r W^{\prime}+\left(4-r \lambda^{\prime}\right) W\right] Y_{l m}, \\
T_{2} \partial_{\theta} Y_{l m} & \equiv r^{3} \delta \pi_{r}^{\theta}=-\check{\mu}\left(r V^{\prime}-2 V+e^{\lambda} W\right) \partial_{\theta} Y_{l m} .
\end{aligned}
$$

These functions, which vanish in the fluid, will help us to apply the boundary conditions for the problem. 
Due to the introduction of the elastic crust, the perturbation equations become more complicated when compared to the fluid case. However, some of the perturbed Einstein equations remain unchanged. Since $\delta \pi_{t}^{b}=0$, the $[t t]$ component provides

$$
\begin{aligned}
e^{-\lambda} r^{2} K^{\prime \prime}+ & e^{-\lambda}\left(3-\frac{1}{2} r \lambda^{\prime}\right) r K^{\prime}-\left[\frac{1}{2} l(l+1)-1\right] K \\
& -e^{-\lambda} r H_{2}^{\prime}-\left[\frac{1}{2} l(l+1)+e^{-\lambda}\left(1-r \lambda^{\prime}\right)\right] H_{2}=-8 \pi r^{2} \delta \rho .
\end{aligned}
$$

Because $\delta \pi_{a}{ }^{b}$ is traceless, we can take the trace of the perturbed Einstein equations to obtain another equation that has no explicit dependence on the elasticity. We combine the trace with (29a) to obtain

$$
\begin{aligned}
& -r^{2} H_{0}^{\prime \prime}+\left[r\left(\frac{1}{2} \lambda^{\prime}-\nu^{\prime}\right)-2\right] r H_{0}^{\prime}+l(l+1) e^{\lambda} H_{0} \\
& -\frac{1}{2} r^{2} \nu^{\prime} H_{2}^{\prime}+\left[2\left(e^{\lambda}-1\right)-r\left(3 \nu^{\prime}+\lambda^{\prime}\right)\right] H_{2}+r^{2} \nu^{\prime} K^{\prime}=8 \pi r^{2} e^{\lambda}(\delta \rho+3 \delta p) .
\end{aligned}
$$

Furthermore, we find from the $[t r]$ component that, as in the fluid case, $H_{1}$ vanishes.

Now, we consider the non-zero components of $\delta \pi_{a}{ }^{b}$. The difference between the $[\theta \theta]$ and $[\phi \phi]$ components leads to the algebraic relation

$$
H_{2}-H_{0}=32 \pi \check{\mu} V
$$

which will be useful to eliminate $H_{2}$ from our equations. We can use the $[r \theta]$ component and (29c) to provide

$$
K^{\prime}=H_{0}^{\prime}+\nu^{\prime} H_{0}+\frac{16 \pi}{r}\left(2+r \nu^{\prime}\right) \check{\mu} V-\frac{16 \pi}{r} T_{2} .
$$

The sum of the $[\theta \theta]$ and $[\phi \phi]$ components gives

$$
\begin{aligned}
\delta p=\frac{e^{-\lambda}\left(\nu^{\prime}+\lambda^{\prime}\right)}{16 \pi r} H_{0}+\frac{e^{-\lambda}}{r^{2}}\left\{e^{\lambda}[2-l(l+1)] \check{\mu} V\right. \\
\left.+\frac{e^{\lambda}}{2} T_{1}-r T_{2}^{\prime}-\left[\frac{1}{2} r\left(\nu^{\prime}-\lambda^{\prime}\right)+1\right] T_{2}\right\},
\end{aligned}
$$

where we have simplified using (29c) and (29d). The final equation we will use from the perturbed Einstein equations is the $[r r]$ component combined with Eqs. (29c)-(29e):

$$
\begin{aligned}
{[l(l+1)-2] e^{\lambda} K=} & r^{2} \nu^{\prime} H_{0}^{\prime}+\left[l(l+1) e^{\lambda}-2-r\left(\nu^{\prime}+\lambda^{\prime}\right)+r^{2} \nu^{\prime 2}\right] H_{0} \\
& +16 \pi\left\{[l(l+1)-2] e^{\lambda}+r^{2} \nu^{\prime 2}\right\} \check{\mu} V \\
& -24 \pi e^{\lambda} T_{1}+16 \pi r T_{2}^{\prime}-8 \pi\left[2+r\left(\nu^{\prime}+\lambda^{\prime}\right)\right] T_{2} .
\end{aligned}
$$


When $\check{\mu}=0$, this reduces to (24b), as expected. Note that the corresponding equation in [27] differs from this by missing a factor of $e^{\lambda}$ in the coefficients of $T_{1}$ and $V$.

The next step is to formulate the system of equations in a way that is straightforward to integrate numerically. Clearly, there is a lot of freedom in how one can do this. We choose to work with the functions $\left(H_{0}^{\prime}, H_{0}, K, W, V, T_{2}\right)$ as our integration variables. It is useful to observe that through (29c) one can reduce the order of the system to eliminate $H_{2}$. In contrast to the fluid case, we are able to solve for the components of the displacement vector by using the definitions of the traction variables (28). To be precise, we can integrate

$$
W^{\prime}-\left(\frac{2}{r}-\frac{\lambda^{\prime}}{2}\right) W=\frac{1}{2} r\left(K-H_{0}\right)-\left[16 \pi r \check{\mu}+\frac{l(l+1)}{2 r}\right] V-\frac{3}{4 \check{\mu} r} T_{1}
$$

and

$$
V^{\prime}-\frac{2}{r} V=-\frac{e^{\lambda}}{r} W-\frac{1}{\check{\mu} r} T_{2} .
$$

We obtain an algebraic relation by combining (29e) and (29f) in such a way as to remove $T_{2}^{\prime}$. This gives us an equation which involves $\delta p$ and $T_{1}$,

$$
\begin{aligned}
16 \pi r^{2} e^{\lambda} \delta p= & r^{2} \nu^{\prime} H_{0}^{\prime}+\left[l(l+1) e^{\lambda}-2+r^{2} \nu^{\prime 2}\right] H_{0}+[2-l(l+1)] e^{\lambda} K \\
& +16 \pi r^{2} \nu^{\prime 2} \check{\mu} V-16 \pi e^{\lambda} T_{1}-16 \pi\left(2+r \nu^{\prime}\right) T_{2} .
\end{aligned}
$$

From (29e) we can obtain an equation to integrate for $T_{2}$ :

$$
T_{2}^{\prime}+\left(\frac{\nu^{\prime}-\lambda^{\prime}}{2}+\frac{1}{r}\right) T_{2}=-r e^{\lambda} \delta p+\frac{\nu^{\prime}+\lambda^{\prime}}{16 \pi} H_{0}+\frac{e^{\lambda}}{r}[2-l(l+1)] \check{\mu} V+\frac{e^{\lambda}}{2 r} T_{1} .
$$

We combine Eqs. (29b)-(29d) to get

$$
\begin{aligned}
& H_{0}^{\prime \prime}+\left(\frac{2}{r}+\frac{\nu^{\prime}-\lambda^{\prime}}{2}\right) H_{0}^{\prime}+\left\{\frac{2}{r^{2}}-[2+l(l+1)] \frac{e^{\lambda}}{r^{2}}+\frac{3 \nu^{\prime}+\lambda^{\prime}}{r}-\nu^{\prime 2}\right\} H_{0} \\
& =-8 \pi\left[e^{\lambda}\left(3+\frac{1}{c_{\mathrm{s}}^{2}}\right) \delta p+2 \nu^{\prime}(\check{\mu} V)^{\prime}+8\left(\frac{1-e^{\lambda}}{r^{2}}+\frac{2 \nu^{\prime}+\lambda^{\prime}}{2 r}-\frac{1}{4} \nu^{\prime 2}\right) \check{\mu} V+\frac{2 \nu^{\prime}}{r} T_{2}\right] .
\end{aligned}
$$

In the fluid, where the shear modulus vanishes, one can verify that (30d) and (30e) reduce to give (24a). The final equation we need from the perturbed Einstein equations (29d), usefully, needs no further alteration,

$$
K^{\prime}=H_{0}^{\prime}+\nu^{\prime} H_{0}+\frac{16 \pi}{r}\left(2+r \nu^{\prime}\right) \check{\mu} V-\frac{16 \pi}{r} T_{2} .
$$

To close this system of equations we need to consider the thermodynamics. We can use (21) and (22) to obtain a second algebraic relation involving $\delta p$ and $T_{1}$ by substituting for $W^{\prime}$ in 
$\perp_{\mathrm{g}}(17)$ :

$$
\frac{3}{4 \check{\mu}} T_{1}=\frac{r^{2}}{(\rho+p) c_{\mathrm{s}}^{2}} \delta p+\frac{3}{2} r^{2} K-\frac{3}{2} l(l+1) V+\left(3-\frac{r \nu^{\prime}}{2 c_{\mathrm{s}}^{2}}\right) W .
$$

We use (30c) and (30g) to determine $\delta p$ and $T_{1}$. Eqs. (30) fully specify the elastic perturbation problem.

\section{Boundary conditions}

To solve for the perturbations throughout the star one needs to solve Eqs. (24) in the fluid regions and Eqs. (30) in the crust. At the centre of the star, the equations are singular and so we demand regularity to obtain the initial condition, for small $r$,

$$
H_{0}(r)=a_{0} r^{l}\left[1+\mathcal{O}\left(r^{2}\right)\right]
$$

where $a_{0}$ is a constant. This can be derived by considering a power-series expansion for small $r$. We note that, a priori, we do not know the amplitude of the perturbations since we have not specified the mechanism that sources them. To single out a unique solution from this one-parameter family of solutions one must match the interior solution to the exterior vacuum solution, which, in the case of tidal deformations, is sourced by the tidal potential of the companion star [35].

There are two fluid-elastic interfaces in the neutron star, where one has to consider the continuity of the perturbed variables. From our assumption that the background star is in a relaxed state, we know that the background quantities will be continuous across an interface. Of course, should one use an equation of state that involves discontinuities at such an interface, that would need to be taken into account. We do not consider such possibilities here, but will do so in upcoming work [37].

In order to determine how the perturbed quantities behave at an interface, we must calculate the first and second fundamental forms and demand that they are continuous across the interface. We describe this calculation in detail in Appendix A. The first fundamental form implies that the functions $H_{0}, K$ and $W$ are continuous. From the second fundamental form we obtain continuity of the radial, $\left(T_{1}+r^{2} \delta p\right)$, and tangential traction, $T_{2}$. We will assume that the shear modulus is non-zero throughout the crust and, therefore, must be discontinuous at a fluid-elastic boundary. Alternatively, one could consider a shear modulus that smoothly goes to zero at an interface. In this case, one might assume that the traction 
conditions would be trivially satisfied. This may be more realistic, but it is difficult to model as we do not have a description for the precise core-crust transition.

In the core we calculate $H_{0}^{\prime}, H_{0}$ and $K$, but in the crust the order of the system increases as we need to calculate the additional functions $W, V$ and $T_{2}$. We know that in the fluid the shear modulus vanishes and so $T_{2}=0$ at both the core-crust and crust-ocean interfaces. We can use (A17) along with (24b) to obtain an expression which is true in the elastic crust at an interface,

$$
r^{2} \nu^{\prime} H_{0}^{\prime}=-\left[l(l+1) e^{\lambda}-2-r\left(\nu^{\prime}+\lambda^{\prime}\right)+r^{2} \nu^{\prime 2}\right] H_{0}+[l(l+1)-2] e^{\lambda} K-16 \pi r^{2} \nu^{\prime 2} \check{\mu} V .
$$

With the six boundary conditions - continuity of $H_{0}$ and $K$ at the core-crust interface, and the constraints at both interfaces: $T_{2}=0$ and $(32)-$ the system is well posed as a boundary-value problem.

The surface of the perturbed configuration is defined to be where the Lagrangian variation of the pressure vanishes, $\Delta p=0$. Because of (21), this conveniently coincides with the definition of the surface for the background star, $p=0$. We describe our numerical approach to solving this problem in detail in Appendix B.

\section{TIDAL DEFORMATIONS}

The formalism we have detailed in Sec. II can be applied to a variety of problems, such as tidal deformations and mountains on neutron stars. In this work, we specialise the perturbations to those sourced by tides in binary systems.

\section{A. The tidal deformability}

A star of mass $M$ and radius $R$ in a time-independent, external tidal field $\mathcal{E}_{i j}$ will develop a quadrupole moment $Q_{i j}$ in response. To linear order, one can relate the quadrupole moment to the tidal field by [35]

$$
Q_{i j}=-\frac{2}{3} k_{2} R^{5} \mathcal{E}_{i j}
$$

where we have introduced the tidal Love number $k_{2}$. We briefly review the procedure of calculating the tidal Love number below, closely following the explanation in [35]. For other detailed discussions on the subject we refer the reader to [38, 39]. 
The Love number can be extracted from the asymptotic behaviour of the metric. In asymptotically Cartesian and mass-centred coordinates, one can write [40]

$$
-\frac{1+g_{t t}}{2}=-\frac{M}{r}-\frac{3 Q_{i j}}{2 r^{3}}\left(n^{i} n^{j}-\frac{1}{3} \delta^{i j}\right)+\mathcal{O}\left(1 / r^{4}\right)+\frac{1}{2} r^{2} \mathcal{E}_{i j} n^{i} n^{j}+\mathcal{O}\left(r^{3}\right),
$$

where $x^{i}$ is the vector that points from the origin to $r, n^{i}=x^{i} / r$ is the corresponding unit vector and $g_{a b}=g_{a b}+h_{a b}$ corresponds to the full metric up to first order. In the vacuum exterior, one should note that $\nu=-\lambda$, therefore, (24a) reduces to

$$
H_{0}^{\prime \prime}+\left(\frac{2}{r}-\lambda^{\prime}\right) H_{0}^{\prime}-\left[l(l+1) \frac{e^{\lambda}}{r^{2}}+\lambda^{\prime 2}\right] H_{0}=0 .
$$

The solution to (35) may be expressed in terms of the associated Legendre polynomials $\mathcal{Q}_{\alpha \beta}(r / M-1)$ and $\mathcal{P}_{\alpha \beta}(r / M-1)$ with $\alpha=l, \beta=2$,

$$
H_{0}(r)=c_{1} \mathcal{Q}_{l 2}(r / M-1)+c_{2} \mathcal{P}_{l 2}(r / M-1)
$$

which gives, when we specialise to quadrupolar $(l=2)$ perturbations,

$$
\begin{aligned}
H_{0}(r)=c_{1}\left(\frac{r}{M}\right)^{2}\left(1-\frac{2 M}{r}\right)[ & -\frac{M(M-r)\left(2 M^{2}+6 M r-3 r^{2}\right)}{r^{2}(2 M-r)^{2}} \\
& \left.+\frac{3}{2} \ln \left(\frac{r}{r-2 M}\right)\right]+3 c_{2}\left(\frac{r}{M}\right)^{2}\left(1-\frac{2 M}{r}\right),
\end{aligned}
$$

where $c_{1}$ and $c_{2}$ are constants to be determined. The asymptotic behaviour of (37) is

$$
H_{0}(r)=\frac{8}{5}\left(\frac{M}{r}\right)^{3} c_{1}+\mathcal{O}\left[(M / r)^{4}\right]+3\left(\frac{r}{M}\right)^{2} c_{2}+\mathcal{O}(r / M) .
$$

One can decompose the tensor multipole moments as

$$
\begin{aligned}
\mathcal{E}_{i j} & =\sum_{m=-2}^{2} \mathcal{E}_{2 m} \mathcal{Y}_{i j}^{2 m}, \\
Q_{i j} & =\sum_{m=-2}^{2} Q_{2 m} \mathcal{Y}_{i j}^{2 m},
\end{aligned}
$$

where the symmetric, trace-free tensors $\mathcal{Y}_{i j}^{l m}$ are defined by [40]

$$
Y_{l m}=\mathcal{Y}_{i j}^{l m} n^{i} n^{j}
$$

One is free to assume that only one $\mathcal{E}_{2 m}$ is non-vanishing, without any loss of generality. Making use of the decomposition of the multipole moments (39), one can insert (38) into 
(34) to show

$$
\begin{aligned}
c_{1} & =\frac{15}{8} \frac{1}{M^{3}} Q_{2 m}, \\
c_{2} & =-\frac{1}{3} M^{2} \mathcal{E}_{2 m}
\end{aligned}
$$

and, thus, obtain

$$
\frac{c_{1}}{c_{2}}=\frac{15}{4} \frac{k_{2}}{C^{5}}
$$

where $C \equiv M / R$ is the star's compactness. Because $H_{0}$ and $H_{0}^{\prime}$ are continuous between the interior and the vacuum at the surface, we can use (37) to determine the ratio $c_{1} / c_{2}$ in terms of the interior solutions at $r=R$. This gives the result [35]

$$
\begin{aligned}
k_{2}=\frac{8 C^{5}}{5}(1 & -2 C)^{2}[2+2 C(y-1)-y]\{2 C[6-3 y+3 C(5 y-8)] \\
& +4 C^{3}\left[13-11 y+C(3 y-2)+2 C^{2}(1+y)\right] \\
& \left.+3(1-2 C)^{2}[2-y+2 C(y-1)] \ln (1-2 C)\right\}^{-1},
\end{aligned}
$$

where we have introduced the parameter $y \equiv R H_{0}^{\prime}(R) / H_{0}(R)$. It is interesting to note that for the computation of the Love number the amplitude $a_{0}$ in the initial condition (31) may be chosen freely. The reason for this is intuitive. Since the tidal Love number is a measure of how deformable a star is in the presence of a quadrupolar field, it is independent of the exact details of an external field and, therefore, the calculation of this quantity is insensitive to the magnitude. We see this in (43) as the ratio $y$ means that dependence on $a_{0}$ exactly cancels. For our analysis, we will focus on the dimensionless tidal deformability parameter,

$$
\Lambda=\frac{2}{3} \frac{k_{2}}{C^{5}}
$$

to enable direct comparison with gravitational-wave constraints (see, e.g., [21]).

Note that the expression (43) is derived under the assumption that $H_{0}^{\prime}$ is continuous across the surface. This is contingent on the final stellar layer having a vanishing shear modulus, or equivalently being a fluid (A17). This detail was overlooked by [28], who treat the outer layer to be the elastic crust and yet use (43) to calculate the tidal Love number. One could of course compute the Love number for a star with an elastic outer region, however, one would need to incorporate the discontinuity of $H_{0}^{\prime}$ using (A17) by taking into account the value of $V$ at the surface. 
We wish to quantify the impact an elastic crust has on tidal deformations. To accurately prescribe the crust-ocean transition, we consider the melting point of the crust. The Coulomb lattice melts when the thermal energy,

$$
E_{\mathrm{th}}=k_{\mathrm{B}} T
$$

where $k_{\mathrm{B}}$ is Boltzmann's constant and $T$ is the temperature, exceeds the interaction energy of the lattice,

$$
E_{\text {Coul }}=\frac{1}{4 \pi \varepsilon_{0}} \frac{Z^{2} e^{2}}{a}
$$

where $Z$ is the proton number, $e$ is the unit charge, $a$ is the mean spacing between nuclei and $\varepsilon_{0}$ is the permittivity of free space, by a critical factor $1 / \Gamma$ :

$$
E_{\text {th }} \geq \frac{1}{\Gamma} E_{\text {Coul }}
$$

where $\Gamma \approx 173$. We assume that the crust forms a body-centred cubic lattice, which has two nuclei per unit cube, so given the number density of nuclei, $n_{\mathrm{N}}$, we have

$$
n_{\mathrm{N}} a^{3}=2 .
$$

The density at which the crust begins to melt is, therefore, obtained from

$$
\rho_{\text {top }}=A m_{\mathrm{u}} n_{\mathrm{N}}=2 A m_{\mathrm{u}}\left(4 \pi \varepsilon_{0} \frac{\Gamma k_{\mathrm{B}} T}{Z^{2} e^{2}}\right)^{3} \approx 6.72 \times 10^{5}\left(\frac{A}{56}\right)\left(\frac{Z}{26}\right)^{-2 / 3}\left(\frac{T}{10^{7} \mathrm{~K}}\right)^{3} \mathrm{~g} \mathrm{~cm}^{-3},
$$

where $A$ is the nucleon number and $m_{\mathrm{u}}$ is the atomic mass unit. For our prescription, we assume that the outer parts of the crust are composed of iron, $Z=26$ and $A=56$, and a temperature of $T=10^{7} \mathrm{~K}$.

The star is defined to have: (i) a fluid core for $\rho_{\mathrm{c}} \geq \rho>\rho_{\text {base }}$, (ii) an elastic crust in the region $\rho_{\text {base }} \geq \rho>\rho_{\text {top }}$ and (iii) a fluid ocean for $\rho \leq \rho_{\text {top }}$, where the base of the crust is defined to be $\rho_{\text {base }}=1.3 \times 10^{14} \mathrm{~g} \mathrm{~cm}^{-3}$. We use the BSk20 analytic equation of state [41] for the high-density fluid core and the equation-of-state table from [42] for the low-density regions. We parametrise each stellar model according to its central density and integrate Eqs. (6) for the background. The background is solved along with Eqs. (24) in the fluid regions of the star and Eqs. (30) in the crust. The results of the integrations are summarised in Table I. The mass and radius of each stellar model is presented in Fig. 1 to show that they are all stable to radial perturbations. 
TABLE I. Results of the numerical integrations of the perturbation equations using the BSk20 equation of state for the core [41] and the equation of state from [42] for the low-density layers of the star. Each stellar model is determined by the central density $\rho_{\mathrm{c}}$. We provide the radius $R$, mass $M$, compactness $C$ and crustal thickness $\Delta R_{\mathrm{c}}$ for each star. The tidal deformability for the fluid stars, $\Lambda_{\text {fluid }}$, and those with elastic crusts, $\Lambda_{\text {crust }}$, are shown, along with the relative difference between them, where $\Delta \Lambda \equiv \Lambda_{\text {crust }}-\Lambda_{\text {fluid }}$. From the differences between the tidal deformabilities, we see that the correction due to the presence of a crust is very small.

\begin{tabular}{|c|c|c|c|c|c|c|c|}
\hline$\rho_{\mathrm{c}} / 10^{15} \mathrm{~g} \mathrm{~cm}^{-3}$ & $R / \mathrm{km}$ & $M / M_{\odot}$ & $C$ & $\Lambda_{\text {crust }}$ & $\Lambda_{\text {fluid }}$ & $\Delta \Lambda / \Lambda_{\text {fluid }}$ & $\Delta R_{\mathrm{c}} / \mathrm{km}$ \\
\hline 2.500 & 10.309 & 2.162 & 0.310 & 3.95452368861 & 3.95452375613 & $-1.707 \times 10^{-8}$ & 0.278 \\
\hline 2.203 & 10.548 & 2.146 & 0.301 & 5.47304743630 & 5.47304753879 & $-1.873 \times 10^{-8}$ & 0.307 \\
\hline 1.941 & 10.787 & 2.112 & 0.289 & 8.01552675384 & 8.01552692367 & $-2.119 \times 10^{-8}$ & 0.343 \\
\hline 1.710 & 11.019 & 2.056 & 0.276 & 12.49881579778 & 12.49881610913 & $-2.491 \times 10^{-8}$ & 0.391 \\
\hline 1.507 & 11.234 & 1.974 & 0.260 & 20.87848456088 & 20.87848520087 & $-3.065 \times 10^{-8}$ & 0.451 \\
\hline 1.327 & 11.423 & 1.864 & 0.241 & 37.57928548491 & 37.57928697864 & $-3.975 \times 10^{-8}$ & 0.529 \\
\hline 1.170 & 11.576 & 1.725 & 0.220 & 73.25641923536 & 73.25642323791 & $-5.464 \times 10^{-8}$ & 0.629 \\
\hline 1.031 & 11.686 & 1.560 & 0.197 & 155.28383741339 & 155.28384983242 & $-7.998 \times 10^{-8}$ & 0.758 \\
\hline 0.908 & 11.748 & 1.375 & 0.173 & 358.77773415782 & 358.77777902699 & $-1.251 \times 10^{-7}$ & 0.926 \\
\hline 0.800 & 11.768 & 1.178 & 0.148 & 903.80359991409 & 903.80378919034 & $-2.094 \times 10^{-7}$ & 1.144 \\
\hline
\end{tabular}

For each stellar model, we compute the tidal deformability in the presence of an elastic crust, $\Lambda_{\text {crust }}$, as well when a crust is not present, $\Lambda_{\text {fluid }}$, for comparison. We also calculate the thickness of the crust, $\Delta R_{\mathrm{c}}$. We show these quantities in Fig. 2 against the central density. In agreement with [27], we find that the inclusion of an elastic crust has an almost negligible impact on the tidal deformability - the correction is the largest for the least compact stars at around two parts in $10^{7}$. This is because, as the compactness decreases, the crust takes up a much larger fraction of the star. Moreover, as one would expect, the crust works to resist the star's deformation which is why the tidal deformabilities computed with a crust are smaller.

To facilitate direct comparison with [27] we also integrated the perturbation equations with a polytropic equation of state and a shear modulus that scales linearly with the pressure. We used the same parameters as [27] and moved the core-crust transition to 


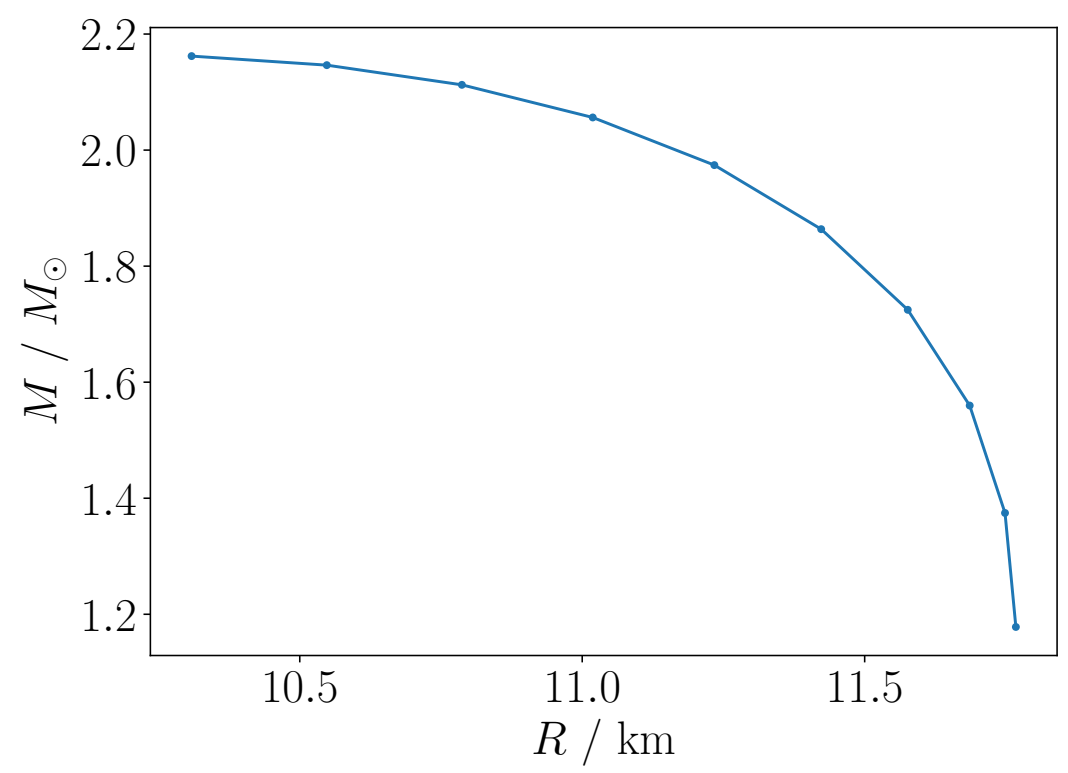

FIG. 1. The mass-radius diagram for the central densities considered, showing that the stellar models considered are stable to radial perturbations.

$\rho_{\text {base }}=2 \times 10^{14} \mathrm{~g} \mathrm{~cm}^{-3}$ and the crust-ocean transition to $\rho_{\text {top }}=10^{7} \mathrm{~g} \mathrm{~cm}^{-3}$. The result is shown in Fig. 3. In our calculation, we find that the tidal deformability is approximately an order of magnitude less sensitive to the inclusion of an elastic crust than reported by [27]. This quantifies the effect of the error from (29f). (It is interesting to note that the crust has a more significant effect in this simple model as compared to the results from the realistic equation of state.)

Furthermore, we note that our results are in stark contrast with those of [28] who find that the crust can make corrections to the tidal deformability of the order of $\sim 1 \%$. The reason for this disagreement is twofold. Firstly, [28] write down expressions for the components of the displacement vector in the fluid and, thus, supposedly compute them in the fluid. This enables them to treat the system of coupled ordinary differential equations as an initial-value problem for the entire star and they use the continuity of the traction in order to match the fluid and elastic regions. As we noted in Sec. II B, due to the static nature of the problem, extracting equations for the components of the displacement vector in the fluid is impossible. Additionally, by computing the perturbations as an initial-value problem means that one does not have the necessary freedom to enforce the traction conditions to be satisfied at the 

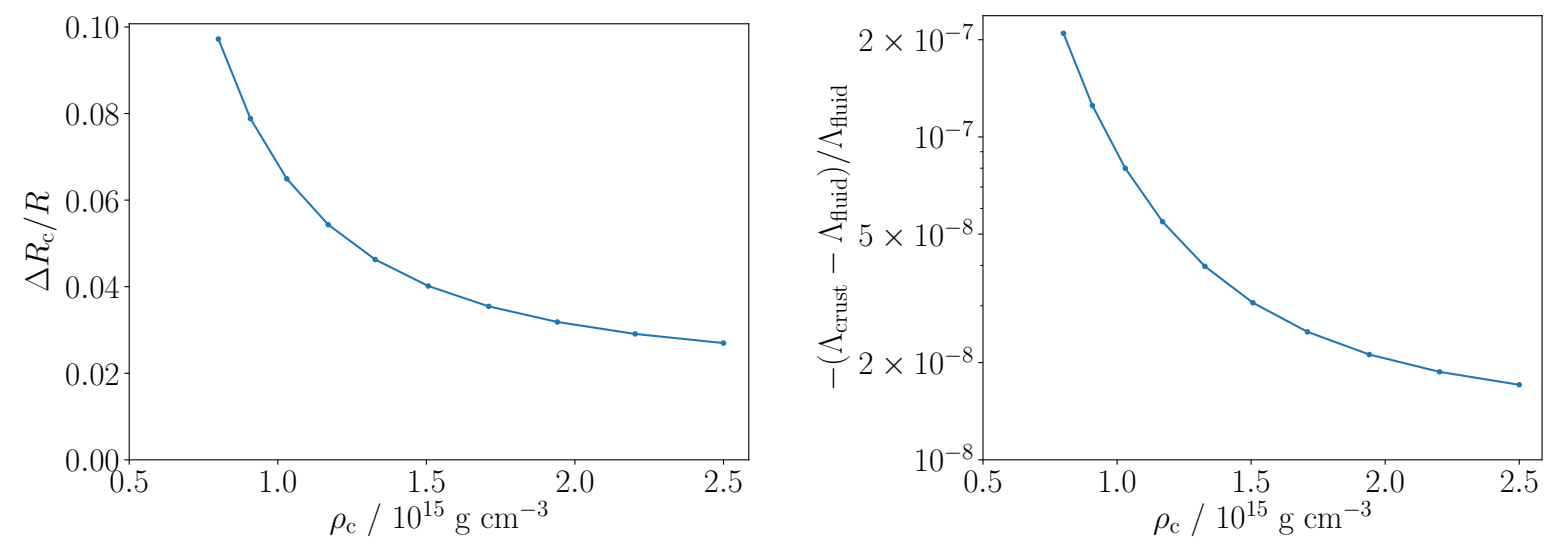

FIG. 2. The ratio of crustal thickness to stellar radius (left panel) and the relative change in tidal deformability due to the presence of a crust (right panel) as functions of central density. As the central density approaches the core-crust transition (which occurs at $\rho_{\text {base }}=1.3 \times 10^{14} \mathrm{~g} \mathrm{~cm}^{-3}$ ) the crust occupies a much larger fraction of the star, so both quantities become more significant.

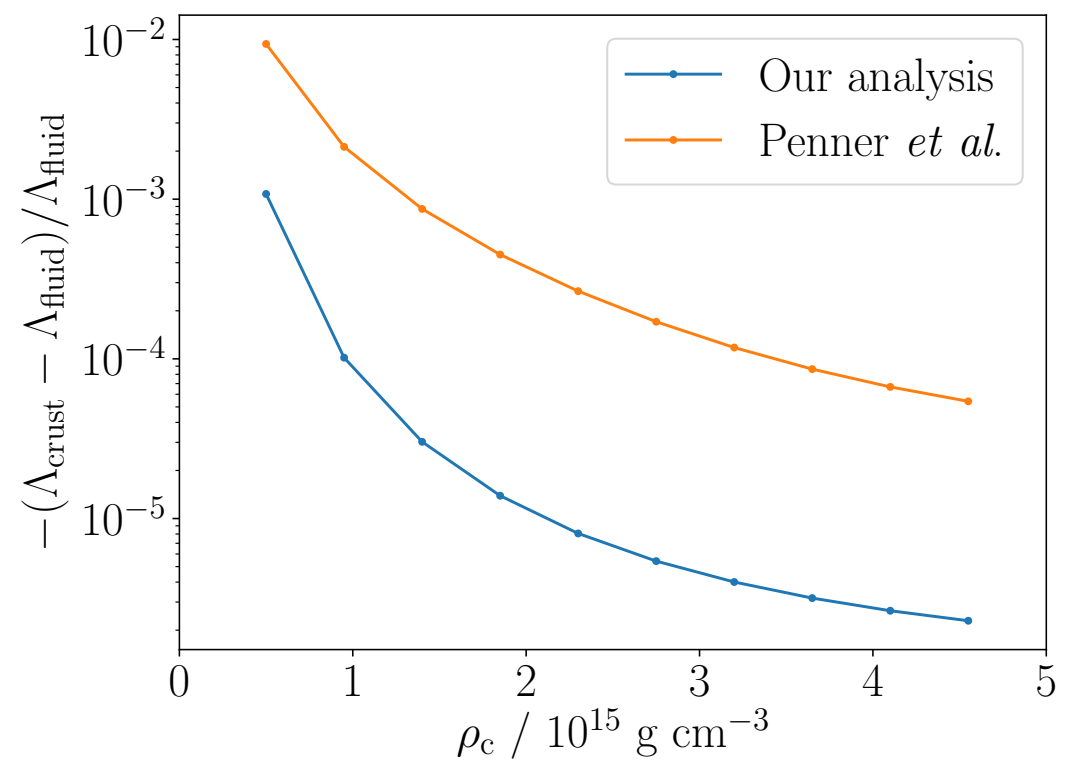

FIG. 3. The relative change in the tidal deformability due to the presence of a crust against the central density for a polytropic equation of state with a linear shear modulus. We compare our results (blue) with those of [27] (orange). 
top of the crust, since the boundary conditions at the centre and the continuity conditions at the core-crust interface are sufficient to carry out the integrations. The second reason is due to the fact that [28] do not have an outer fluid ocean in their stellar model, but instead have an exposed crust. In such a model, $H_{0}^{\prime}$ is discontinuous and, therefore, one cannot use (43) as they do in order to compute the Love number. However, we note that the shear modulus at the top of the crust is expected to be small and so the discontinuity in $H_{0}^{\prime}$ will be small. The difference in these results is important. If one assumes that third-generation gravitational-wave detectors will be able to constrain $\Lambda$ to within a few percent [43], then our results show that the effect of the crust will not be measurable, which is at odds with the results of [28].

\section{B. Crustal failure}

The formalism above allows us to calculate the interior structure of a neutron star with an elastic crust that is experiencing static, even-parity perturbations. We can apply this formalism to determine when and where the crust will begin to fracture during a binary neutron star inspiral, as was done in [44]. In contrast to the computation of the tidal Love number, the amplitude of the perturbations is important for this calculation. Therefore, we must normalise our perturbations by matching the interior solution to the exterior at the surface, and, thus, constrain the amplitude.

We consider a binary separated by distance $d$ where the companion star is of mass $M_{\text {comp }}$. We assume $d \gg r$, as is appropriate in the adiabatic regime, and work in the Newtonian limit for the normalisation. By Kepler's third law, the angular frequency of the binary $\Omega$ is given by

$$
\Omega^{2}=\frac{M+M_{\text {comp }}}{d^{3}}
$$

This is related to the orbital frequency of the binary $f_{\text {orbit }}$ by $\Omega=2 \pi f_{\text {orbit }}$. First, let us estimate the gravitational-wave frequency at merger for an equal-mass binary, $M=M_{\text {comp }}$. We assume that the point of merger corresponds to when the two stars touch, $d=2 R$, and since gravitational waves radiate at twice the orbital frequency, $f_{\mathrm{GW}}=2 f_{\text {orbit }}$, we find

$$
f_{\mathrm{GW}}^{\text {merger }}=\frac{1}{2 \pi} \sqrt{\frac{M}{R^{3}}} \approx 2170\left(\frac{M}{1.4 M_{\odot}}\right)^{1 / 2}\left(\frac{R}{10 \mathrm{~km}}\right)^{-3 / 2} \mathrm{~Hz} .
$$


Expanding around $r=0$ one can show that the external field due to the presence of the companion is

$$
\Phi_{\text {ext }}\left(x^{i}\right)=-\frac{M_{\mathrm{comp}}}{d}-\frac{M_{\mathrm{comp}}}{d^{2}} r m_{i} n^{i}-\frac{3}{2} \frac{M_{\mathrm{comp}}}{d^{3}} r^{2}\left(m_{i} m_{j}-\frac{1}{3} \delta_{i j}\right) n^{i} n^{j},
$$

where $m^{i}$ is the unit vector that points from the centre of the star to the centre of the companion. The tidal piece can be expressed using the $l=2, m=0$ spherical harmonic,

$$
\Phi_{\text {tidal }}\left(x^{i}\right)=-\sqrt{\frac{4 \pi}{5}} \frac{M_{\text {comp }}}{d^{3}} r^{2} Y_{20}
$$

This means that the binary is orientated such that $\theta=0$ points in the direction of $m^{i}$. The tidal multipole in the Newtonian limit is given by, using (52),

$$
\mathcal{E}_{i j}=\frac{\partial^{2} \Phi_{\mathrm{ext}}}{\partial x^{i} \partial x^{j}}=-3 \frac{M_{\mathrm{comp}}}{d^{3}}\left(m_{i} m_{j}-\frac{1}{3} \delta_{i j}\right) .
$$

Using the decomposition of (39) one can show that the non-vanishing $\mathcal{E}_{2 m}$ is

$$
\mathcal{E}_{20}=-2 \sqrt{\frac{4 \pi}{5}} \frac{M_{\mathrm{comp}}}{d^{3}}
$$

and, therefore, by (41b) we find

$$
c_{2}=\frac{2}{3} \sqrt{\frac{4 \pi}{5}} \frac{M^{2} M_{\mathrm{comp}}}{d^{3}}=\frac{2 \pi^{2}}{3} \sqrt{\frac{4 \pi}{5}} \frac{M^{2} M_{\mathrm{comp}}}{M+M_{\mathrm{comp}}} f_{\mathrm{GW}}^{2} .
$$

Here we have chosen to parametrise the point in the inspiral by the gravitational-wave frequency over the separation by using (50). Eqs. (42) and (56) provide the necessary information to normalise the perturbations to a binary that is emitting gravitational waves with frequency $f_{\mathrm{GW}}$.

We use the von Mises criterion to determine when the crust begins to break. In the formalism of [36], one can calculate the von Mises strain for a neutron star, where the unperturbed configuration is unstrained, through

$$
\Theta=\sqrt{\frac{3}{2} \Delta s_{a b} \Delta s^{a b}}
$$

The crust fractures when the von Mises strain reaches the threshold yield point $\Theta \geq \Theta^{\text {break }}$. Using the definition of the traction variables (28) with (26) and specialising to $l=2, m=0$ perturbations, one finds

$$
\Theta^{2}=\frac{45}{256 \pi} \frac{1}{r^{4}}\left[\left(3 \cos ^{2} \theta-1\right)^{2}\left(\frac{T_{1}}{\check{\mu}}\right)^{2}+12 e^{-\lambda} \sin ^{2}(2 \theta)\left(\frac{T_{2}}{\check{\mu}}\right)^{2}+48 \sin ^{4} \theta V^{2}\right]
$$




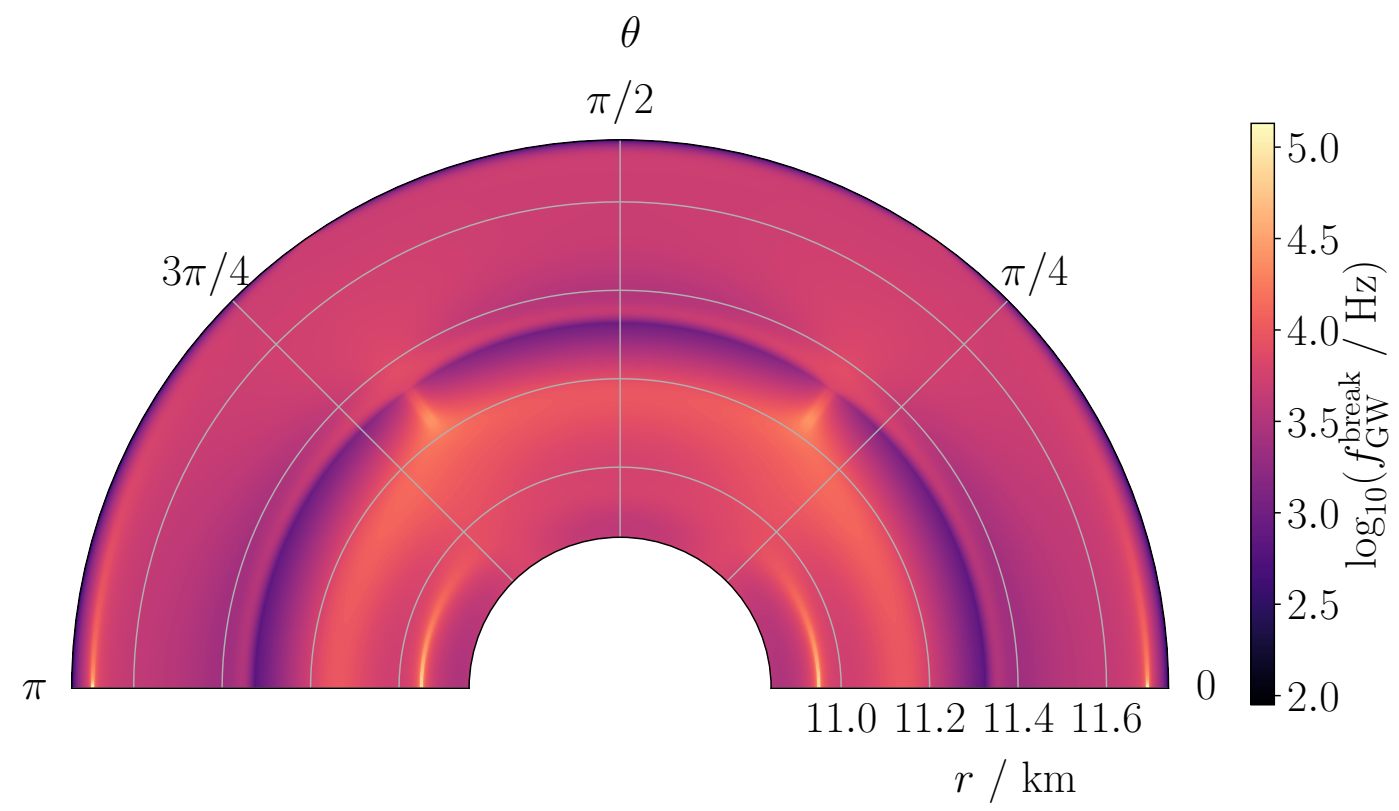

FIG. 4. The gravitational-wave frequency at failure across the elastic crust. We can see that the majority of the crust will fail before merger.

The advantage of using the von Mises strain is that it is a function of position and so we can identify where the crust is the weakest as well as when it breaks. Taking the breaking strain to be $\Theta^{\text {break }}=0.1$ [45], we can calculate when the crust will break, at each point, by imposing that the strain in (58) is equal to $\Theta^{\text {break }}$ to normalise the perturbations and then determining the gravitational-wave frequency $f_{\mathrm{GW}}^{\text {break }}$ which corresponds to that amplitude using (42) and (56).

As an illustration, we use the same equation of state as in Sec. III A. We assume the binary is equal mass with $M=M_{\text {comp }}=1.4 M_{\odot}$, for which we obtain a star with radius $R=11.74 \mathrm{~km}$. In Fig. 4 we show the gravitational-wave frequency when the crust breaks at each point. Fig. 5 focuses on the regions of the star that break before merger. There is a clear phase transition at neutron drip (around $r=11.3 \mathrm{~km}$ ), where the inner crust is, on average, stronger than the outer crust. The crust is notably strong at neutron drip close 


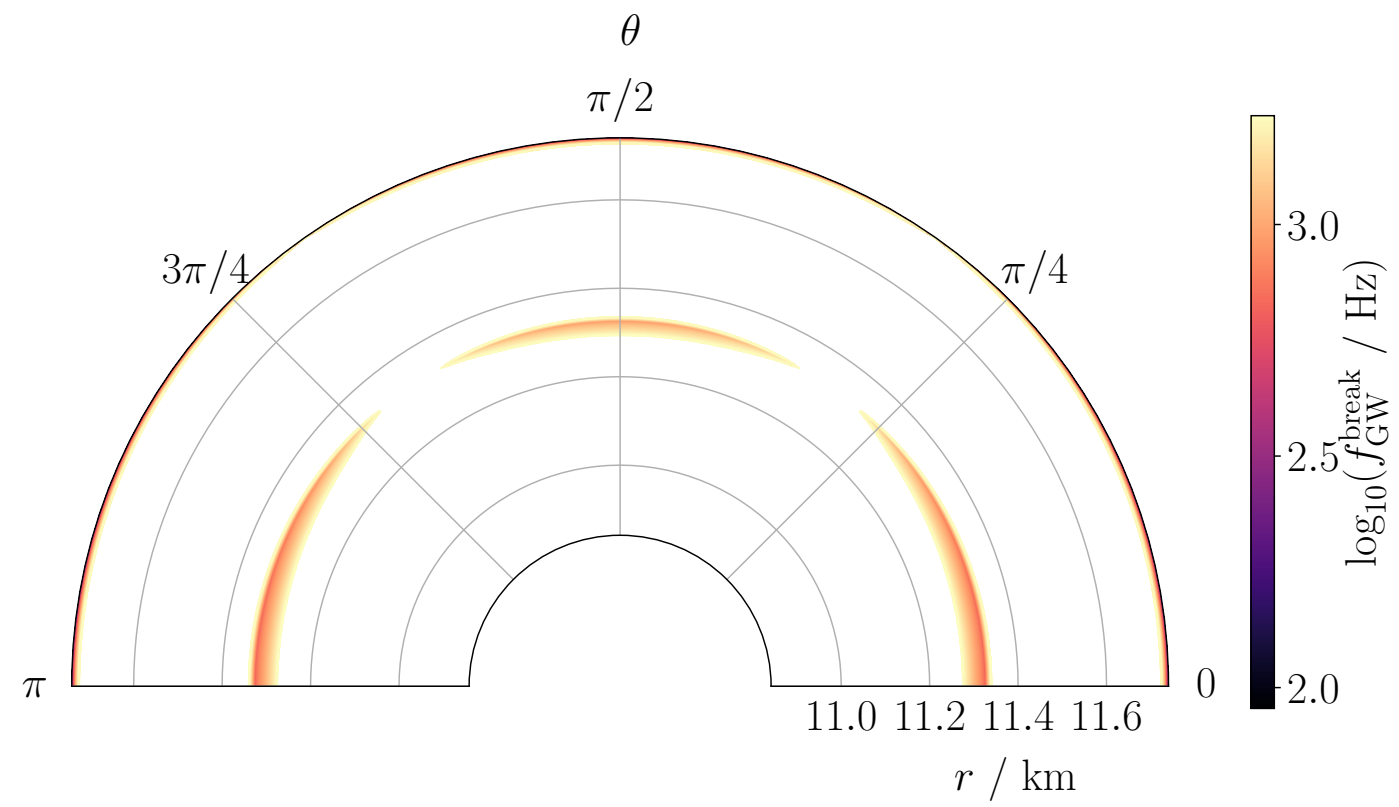

FIG. 5. The gravitational-wave frequency at failure for the locations in the elastic crust that yield before merger. This shows that the stars will merge with the crust largely intact.

to $\theta \approx \pi / 4$ and $3 \pi / 4$. The reason for this is, as the star becomes more oblate, the parts closest to the poles and equator are stretched the most. The region that stretches the least is in between these two regions at $\theta \approx \pi / 4$ and $3 \pi / 4$. This effect can be seen in Figs. 6 and 7 where the tangential functions $T_{2} / \check{\mu}$ and $V$ combine to give a local minimum in the von Mises strain at these angles, and thus a local maximum in the breaking frequency. The maxima along the equator, $\theta=0$ and $\pi$, are where the magnitude of the radial traction function $T_{1} / \check{\mu}$ reaches a local minimum (as shown in Fig. 7).

We note that, as compared to typical merger frequencies (51), our results suggest that the vast majority of the crust will not fracture before merger. In fact, the crust will only fail at neutron drip and in the very outermost part of the outer crust before coalescence. This is in contrast to the results of [44], who obtain significantly lower breaking frequencies throughout the crust. This is likely related to the errors in the analysis of [27], that we 


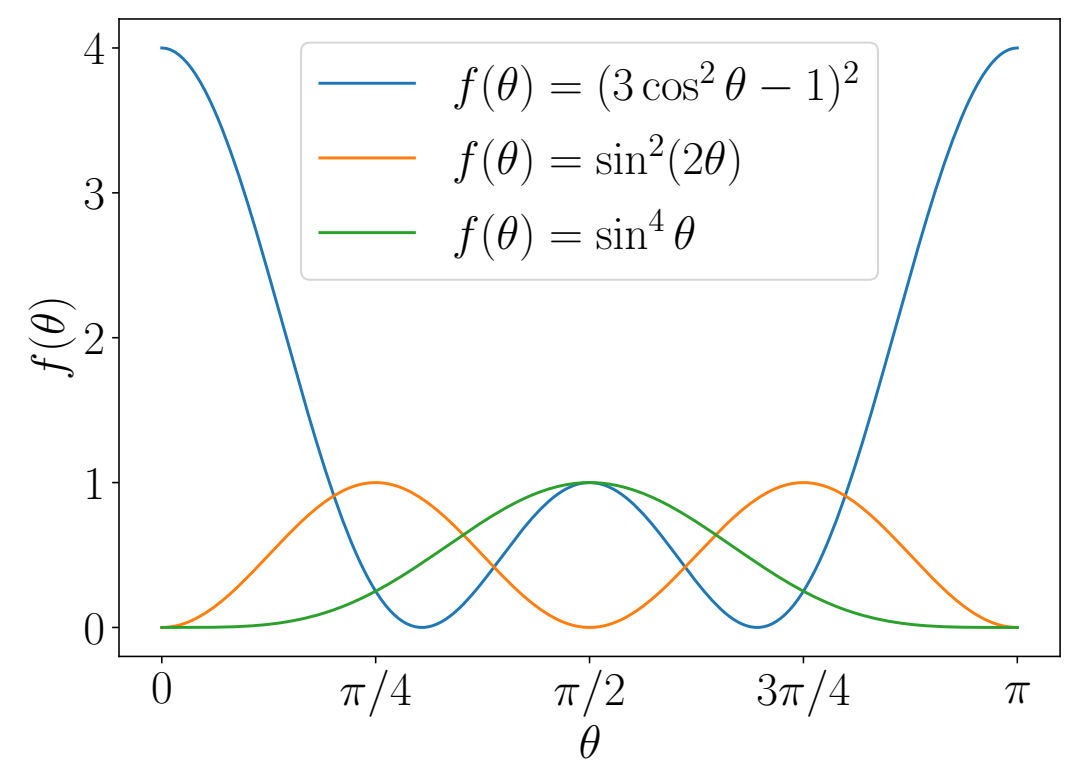

FIG. 6. The angular basis of the von Mises strain for $l=2, m=0$ perturbations.

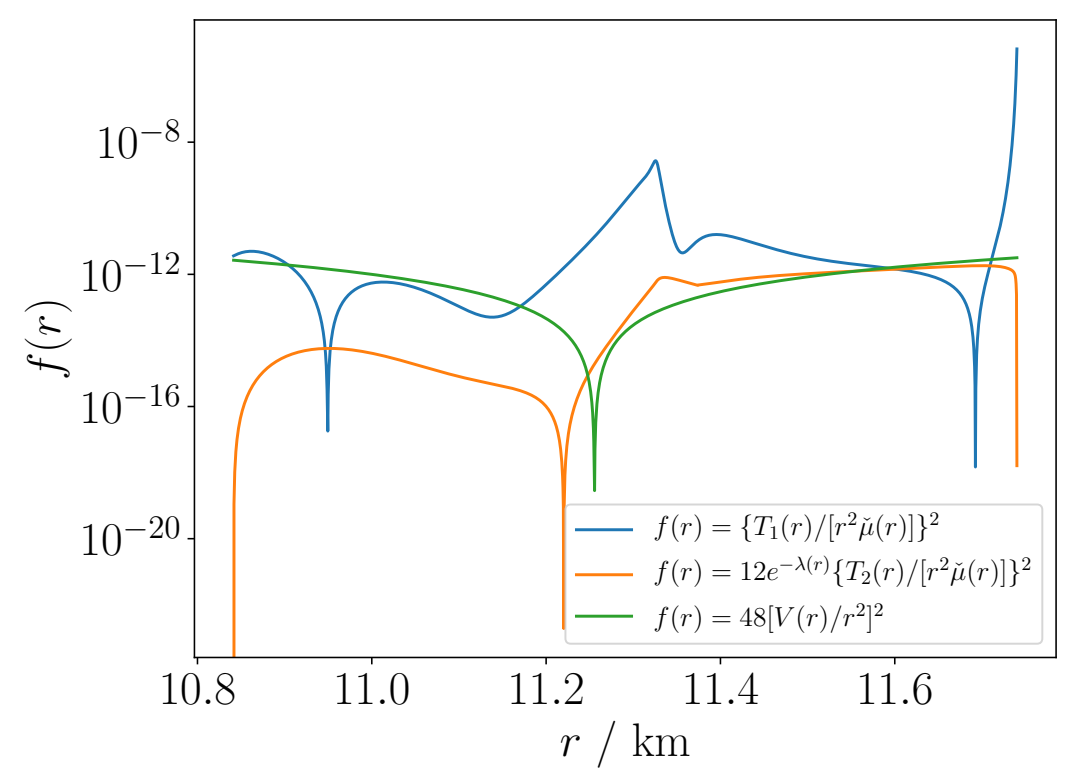

FIG. 7. The radial dependence of the radial and tangential traction variables and the tangential displacement function normalised to a binary radiating gravitational waves with $f_{\mathrm{GW}}=10 \mathrm{~Hz}$. At neutron drip, around $r=11.3 \mathrm{~km}$, we find that the tangential functions combine to give local minima for most values of $\theta$. Note that the vertical axis is in logrithmic scale - the cusps correspond to when the functions cross zero and change sign. 
have pointed out above. Since the crust will be mostly intact by the point of merger, this suggests that there will not be a significant amount of strain energy released available for an associated electromagnetic signal.

\section{CONCLUSIONS}

With the advent of gravitational-wave detections of binary neutron star mergers, we have a promising new method of constraining the equation of state of nuclear matter. The gravitational waveforms from these events are sensitive to tidal effects in the binaries which carry model-independent information on the equation of state.

In this paper, we have explored the impact of an elastic crust on tidal deformations of neutron stars. We have presented a formalism which enables one to compute static, evenparity perturbations of a neutron star with an elastic component. This was necessary to resolve discrepancies between previous studies [27, 28]. There are mistakes in the crustal perturbation equations presented by [27], in particular arising from the analogous equation to (29f). This meant they marginally overestimated the impact of a crust on tidal deformations and consequently this affected their analysis on when the crust will break in a binary inspiral [44]. Meanwhile, the work of [28] calculates the static displacement vector in the fluid regions

of the star. However, such a calculation should not be possible due to the static nature of the problem. This means that they cannot correctly impose continuity of the traction at the top of the crust. Moreover, [28] do not correctly calculate the tidal Love number for the assumed stellar model with an exposed crust.

We have applied our formalism to the computation of static, quadrupolar perturbations of a neutron star sourced by an external tidal field. We calculated the quadrupolar perturbations for a realistic equation of state that includes an elastic crust. We have shown that the inclusion of an elastic crust has a very small effect on the tidal deformability of a star, in the range of $\sim 10^{-8}-10^{-7}$ for realistic models - even smaller than what one would calculate using simplistic equations of state. We found that our results are an order of magnitude smaller than what was reported by [27] and significantly smaller than the results of [28]. This means the impact of a crust on binary neutron star mergers is not expected to be detectable for current and next-generation gravitational-wave detectors.

We used our integrations to calculate when and where the crust would fail during a binary 
inspiral with component masses $M=M_{\text {comp }}=1.4 M_{\odot}$. We found that the crust is much stronger than estimated in previous work [44]. The majority of the crust will not fail before the two neutron stars merge. Only the small regions close to neutron drip and the outer layers of the crust will fracture before merger.

\section{ACKNOWLEDGMENTS}

NA gratefully acknowledges financial support from STFC via grant ST/R00045X/1. JPP is thankful for the partial support by the Polish National Science Centre via grant number 2016/22/E/ST9/00037 and FAPESP via grant numbers 2015/04174-9 and 2017/21384-2.

\section{Appendix A: Calculating the interface conditions}

Since we consider a star with multiple layers that have phase transitions, we must address how the perturbation functions behave across an interface. We calculate the interface conditions using the geometrical approach explained in [46].

Let us begin by considering the level surfaces of a scalar quantity $A$. We assume the level surfaces to be timelike and, therefore, have the normal

$$
\mathcal{N}^{a}=\frac{\partial^{a} A}{\sqrt{\partial^{b} A \partial_{b} A}},
$$

where $\mathcal{N}^{a} \mathcal{N}_{a}=1$ is true by construction. The first fundamental form (also known as the intrinsic curvature or induced three-metric) of these level surfaces is

$$
\gamma_{a b}=P_{a}^{c} P_{b}^{d} g_{c d}
$$

where the projection operator along the level surfaces is given by

$$
P_{a}^{b}=\delta_{a}^{b}-\mathcal{N}_{a} \mathcal{N}^{b}
$$

The second fundamental form (also known as the extrinsic curvature) of the level surfaces is defined as

$$
K_{a b}=-P_{a}^{c} P_{b}^{d} \nabla_{(c} \mathcal{N}_{d)}
$$

Let us specialise and consider the useful decomposition of our scalar quantity of the form

$$
A(t, r, \theta, \phi)=A_{0}(r)+\delta A(t, r, \theta, \phi)
$$


Using this decomposition we obtain the following components for the normal:

$$
\begin{aligned}
\mathcal{N}^{t} & =-e^{-\nu+\lambda / 2} \frac{\partial_{t} \delta A}{A_{0}^{\prime}}+e^{-\nu-\lambda / 2} h_{t r}, \\
\mathcal{N}^{r} & =e^{-\lambda / 2}\left(1-\frac{1}{2} e^{-\lambda} h_{r r}\right), \\
\mathcal{N}^{\theta} & =\frac{e^{\lambda / 2}}{r^{2}} \frac{\partial_{\theta} \delta A}{A_{0}^{\prime}} \\
\mathcal{N}^{\phi} & =\frac{e^{\lambda / 2}}{r^{2} \sin ^{2} \theta} \frac{\partial_{\phi} \delta A}{A_{0}^{\prime}}
\end{aligned}
$$

The level surfaces of $A$, thus, have the following non-zero components of the first fundamental form:

$$
\begin{aligned}
\gamma_{t t} & =-e^{\nu}+h_{t t}, \\
\gamma_{t r} & =h_{t r}-e^{\lambda} \frac{\partial_{t} \delta A}{A_{0}^{\prime}}, \\
\gamma_{r \theta} & =-e^{\lambda} \frac{\partial_{\theta} \delta A}{A_{0}^{\prime}}, \\
\gamma_{r \phi} & =-e^{\lambda} \frac{\partial_{\phi} \delta A}{A_{0}^{\prime}}, \\
\gamma_{\theta \theta} & =r^{2}+h_{\theta \theta}, \\
\gamma_{\phi \phi} & =r^{2} \sin ^{2} \theta+h_{\phi \phi} .
\end{aligned}
$$


The non-trivial components of the second fundamental form are:

$$
\begin{aligned}
K_{t t} & =\frac{\nu^{\prime}}{2} e^{\nu-\lambda / 2}-e^{\lambda / 2} \frac{\partial_{t}^{2} \delta A}{A_{0}^{\prime}}+e^{-\lambda / 2} \partial_{t} h_{t r}-\frac{1}{2} e^{-\lambda / 2} h_{t t}^{\prime}-\frac{\nu^{\prime}}{4} e^{\nu-3 \lambda / 2} h_{r r} \\
K_{t r} & =\frac{\nu^{\prime}}{2}\left(e^{\lambda / 2} \frac{\partial_{t} \delta A}{A_{0}^{\prime}}-e^{-\lambda / 2} h_{t r}\right) \\
K_{t \theta} & =-e^{\lambda / 2}\left(\frac{\partial_{t} \partial_{\theta} \delta A}{A_{0}^{\prime}}-\frac{1}{2} e^{-\lambda} \partial_{\theta} h_{t r}\right) \\
K_{t \phi} & =-e^{\lambda / 2}\left(\frac{\partial_{t} \partial_{\phi} \delta A}{A_{0}^{\prime}}-\frac{1}{2} e^{-\lambda} \partial_{\phi} h_{t r}\right) \\
K_{r \theta} & =\frac{e^{\lambda / 2}}{r} \frac{\partial_{\theta} \delta A}{A_{0}^{\prime}} \\
K_{r \phi} & =\frac{e^{\lambda / 2}}{r} \frac{\partial_{\phi} \delta A}{A_{0}^{\prime}}, \\
K_{\theta \theta} & =-e^{-\lambda / 2} r-e^{\lambda / 2} \frac{\partial_{\theta}^{2} \delta A}{A_{0}^{\prime}}-\frac{1}{2} e^{-\lambda / 2}\left(h_{\theta \theta}^{\prime}-e^{-\lambda} r h_{r r}\right) \\
K_{\theta \phi} & =-\frac{e^{-\lambda / 2}}{A_{0}^{\prime}}\left(\partial_{\theta} \partial_{\phi} \delta A-\cot \theta \partial_{\phi} \delta A\right) \\
K_{\phi \phi} & =-e^{-\lambda / 2} r \sin ^{2} \theta-e^{\lambda / 2} \frac{\partial_{\phi}^{2} \delta A}{A_{0}^{\prime}}-e^{\lambda / 2} \sin \theta \cos \theta \frac{\partial_{\theta} \delta A}{A_{0}^{\prime}}-\frac{1}{2} e^{-\lambda / 2}\left(h_{\phi \phi}^{\prime}-e^{-\lambda} r \sin ^{2} \theta h_{r r}\right)
\end{aligned}
$$

Both the first and second fundamental forms must be continuous across an interface (in the absence of surface degrees of freedom).

As was done by [26], we will consider the level surfaces of the radial shell, so we assign $A_{0}=r$ and $\delta A=\xi^{r}$. We use the perturbed metric for even-parity perturbations (7). Because of how we set up the problem by assuming the background star is in a relaxed state, we know that the background quantities will all be continuous across an interface. We further assume that there is no discontinuity in the density or pressure. The first fundamental form with components $\gamma_{t t}, \gamma_{t r}, \gamma_{\theta \theta}$ and $\gamma_{r \theta}$ show

$$
\left[H_{0}\right]_{r}=0, \quad\left[H_{1}\right]_{r}=0, \quad[K]_{r}=0, \quad\left[\delta A / A_{0}^{\prime}\right]_{r}=0
$$

where we have introduced the notation $[f]_{r}=\lim _{\epsilon \rightarrow 0}[f(r+\epsilon)-f(r-\epsilon)]$ to describe the continuity of a function $f(r)$ at a point $r$. The angular part of $\delta A$ is decomposed using spherical harmonics. For the problem we are analysing, $H_{1}$ simply vanishes. The generic condition above translates to $\left[\xi^{r}\right]_{r}=0$, which is equivalent to

$$
[W]_{r}=0 .
$$


This condition is equivalent to saying there must not be a gap in the perturbed material.

We have exhausted the information we can learn from continuity of the first fundamental form. We also notice that there is no more additional information to be learned from the components $K_{t r}, K_{t \theta}, K_{t \phi}, K_{r \theta}$ and $K_{r \phi}$ of the second fundamental form; only components $K_{t t}$ and $K_{\theta \theta}$ provide more interface conditions. Continuity of $K_{t t}$ implies

$$
\left[h_{t t}^{\prime}+\frac{\nu^{\prime}}{2} e^{\nu-\lambda} h_{r r}\right]_{r}=0
$$

This gives

$$
\left[H_{0}^{\prime}\right]_{r}=-\frac{\nu^{\prime}}{2}\left[H_{2}\right]_{r}
$$

Similarly, we can infer from $K_{\theta \theta}$

$$
\left[h_{\theta \theta}^{\prime}-e^{-\lambda} r h_{r r}\right]_{r}=0 \Rightarrow\left[K^{\prime}\right]_{r}=\frac{1}{r}\left[H_{2}\right]_{r}
$$

We can combine (A12) and (A13) to obtain

$$
\left[K^{\prime}-H_{0}^{\prime}\right]_{r}=\frac{1}{2 r}\left(2+r \nu^{\prime}\right)\left[H_{2}\right]_{r}
$$

Now, we need some information from the perturbed Einstein equations. The above expression can be further used along with (29d) to provide

$$
\frac{16 \pi}{r}\left(2+r \nu^{\prime}\right)[\check{\mu} V]_{r}-\frac{16 \pi}{r}\left[T_{2}\right]_{r}=\frac{1}{2 r}\left(2+r \nu^{\prime}\right)\left[H_{2}\right]_{r} .
$$

Using continuity of $H_{0}$ and (29c) we find

$$
\left[H_{2}\right]_{r}=32 \pi[\check{\mu} V]_{r}
$$

This condition states that we should expect a discontinuity in $H_{2}$ for two reasons: (i) the shear modulus vanishes in the fluid and has a finite value in the crust and (ii) there is no reason that the tangential displacement function $V$ need be continuous. This further implies through (A12) that $H_{0}^{\prime}$ will be discontinuous:

$$
\left[H_{0}^{\prime}\right]_{r}=-16 \pi \nu^{\prime}[\check{\mu} V]_{r}
$$

Eqs. (A15) and (A16) imply continuity of the tangential traction variable

$$
\left[T_{2}\right]_{r}=0
$$


Finally, we use (30c), along with the continuity condition (A17), to obtain

$$
\left[T_{1}+r^{2} \delta p\right]_{r}=0
$$

Since the radial displacement function $W$ is continuous, we can write this in a more general form

$$
\left[T_{1}+r^{2} \Delta p\right]_{r}=0
$$

Eqs. (A18) and (A20) simply mean that the radial and tangential stresses are continuous across a fluid-elastic interface. These interface conditions are necessary when considering how the functions behave across a fluid-elastic boundary and enable one to carry out the integration in the crust.

\section{Appendix B: Numerical scheme}

Our approach to solving the interior perturbation equations is similar to as described in [47] and [48]. We divide our star into three layers: (i) a fluid core from $R_{0}=0$ to $R_{1}$; (ii) an elastic crust from $R_{1}$ to $R_{2}$; and (iii) a fluid ocean from $R_{2}$ to $R_{3}=R$. We express the system of ordinary differential equations for a given layer $i$ in the form

$$
\frac{d \mathbf{Y}^{(i)}}{d r}=\mathbf{Q}^{(i)} \cdot \mathbf{Y}^{(i)}, \text { for } r \in\left[R_{i-1}, R_{i}\right]
$$

where $\mathbf{Y}^{(i)}(r)=\left[y_{1}(r), \ldots, y_{k_{i}}(r)\right]$ is an abstract $k_{i}$-dimensional vector field, $\mathbf{Q}^{(i)}(r)$ is a $k_{i} \times k_{i}$ matrix and $r=R_{i}$ denotes the end of layer $i$. As long as our differential equations are linear we are free to write the system in the above form.

Due to the linearity of the differential equations, we generate a set of $k_{i}$ linearlyindependent solutions $\mathbf{Y}_{j}^{(i)}(r)$ for layer $i$ and obtain the general solution using a linear combination of these solutions,

$$
\mathbf{Y}^{(i)}(r)=\sum_{j=1}^{k_{i}} c_{j}^{(i)} \mathbf{Y}_{j}^{(i)}(r)
$$

where the coefficients $c_{j}^{(i)}$ are constants to be determined from boundary and interface conditions. We generate these linearly-independent solutions by choosing linearly-independent start vectors $\mathbf{Y}_{j}^{(i)}\left(R_{i-1}\right)$ and integrating through the layer using (B1) up to $r=R_{i}$. (Note that, in theory, there is no reason one could not do the reverse, integrating from $r=R_{i}$ to 
$R_{i-1}$, should they wish.) A priori, we do not have any additional information about layer $i$ and would naïvely integrate $k_{i}$ linearly-independent start vectors. However, we can reduce the computational effort by applying relevant boundary conditions. For example, should a variable vanish at an interface, one could simply set this variable to zero in the start vectors and reduce the number of necessary linearly-independent solutions by one.

The fluid regions of the star are governed by Eqs. (24) and so are fully described by the abstract two-dimensional vector field

$$
\mathbf{Y}^{(k)}(r)=\left[H_{0}^{\prime}(r), H_{0}(r)\right]
$$

where $k=1,3$ denotes the core and ocean, respectively. The elastic region of the star is more complex and requires more functions to describe its structure. Thus, we use the six-dimensional vector field

$$
\mathbf{Y}^{(2)}(r)=\left[H_{0}^{\prime}(r), H_{0}(r), K(r), W(r), V(r), T_{2}(r)\right]
$$

At a fluid-elastic interface we know the variables $H_{0}, K, W$ and $T_{2}$ are continuous. We know the values of $H_{0}$ and $K$ from the calculation in the fluid core and so we use their final values in the core to start our integration in the crust. Since the traction variables vanish in the fluid we can simplify the integrations in the elastic by demanding that $T_{2}=0$ at an interface. For each of the solutions we calculate the value for $H_{0}^{\prime}$ at the base using (32). These conditions mean that we must generate two linearly-independent solutions with the initial values for the unknown functions $W$ and $V$. Notice that because of the condition (32) we could equivalently choose to generate solutions with $H_{0}^{\prime}$ instead of $V$. At the top of the crust we demand that $T_{2}=0$ and $H_{0}^{\prime}$ be equal to the expression calculated using (32). We use these two constraints to solve for the coefficients of the general solution. At the top of the crust, we can straightforwardly continue the integration through the fluid ocean, since $H_{0}$ and $K$ are continuous. Although, $H_{0}^{\prime}$ is discontinuous, we can calculate it exactly from $H_{0}$ and $K$ using (24b) and integrate to the surface.

[1] B. P. Abbott et al., GW170817: Observation of Gravitational Waves from a Binary Neutron Star Inspiral, Phys. Rev. Lett. 119, 161101 (2017), arXiv:1710.05832 [gr-qc]. 
[2] B. P. Abbott et al., GW190425: Observation of a Compact Binary Coalescence with Total Mass $\sim 3.4 M_{\odot}$, arXiv e-prints, arXiv:2001.01761 (2020), arXiv:2001.01761 [astro-ph.HE].

[3] A. W. Steiner, S. Gandolfi, F. J. Fattoyev, and W. G. Newton, Using neutron star observations to determine crust thicknesses, moments of inertia, and tidal deformabilities, Phys. Rev. C 91, 015804 (2015), arXiv:1403.7546 [nucl-th].

[4] M. B. Tsang, J. R. Stone, F. Camera, P. Danielewicz, S. Gandolfi, K. Hebeler, C. J. Horowitz, J. Lee, W. G. Lynch, Z. Kohley, R. Lemmon, P. Möller, T. Murakami, S. Riordan, X. RocaMaza, F. Sammarruca, A. W. Steiner, I. Vidaña, and S. J. Yennello, Constraints on the symmetry energy and neutron skins from experiments and theory, Phys. Rev. C 86, 015803 (2012), arXiv:1204.0466 [nucl-ex].

[5] M. Baldo and G. F. Burgio, The nuclear symmetry energy, Prog. Part. Nucl. Phys. 91, 203 (2016), arXiv:1606.08838 [nucl-th].

[6] J. M. Lattimer and M. Prakash, The equation of state of hot, dense matter and neutron stars, Phys. Rep. 621, 127 (2016), arXiv:1512.07820 [astro-ph.SR].

[7] M. Oertel, M. Hempel, T. Klähn, and S. Typel, Equations of state for supernovae and compact stars, Rev. Mod. Phys. 89, 015007 (2017), arXiv:1610.03361 [astro-ph.HE].

[8] J. M. Lattimer and M. Prakash, Neutron star observations: Prognosis for equation of state constraints, Phys. Rep. 442, 109 (2007), arXiv:astro-ph/0612440 [astro-ph].

[9] A. W. Steiner, C. O. Heinke, S. Bogdanov, C. K. Li, W. C. G. Ho, A. Bahramian, and S. Han, Constraining the mass and radius of neutron stars in globular clusters, Mon. Not. R. Astron Soc. 476, 421 (2018), arXiv:1709.05013 [astro-ph.HE].

[10] A. W. Steiner, J. M. Lattimer, and E. F. Brown, The Equation of State from Observed Masses and Radii of Neutron Stars, Astrophys. J. 722, 33 (2010), arXiv:1005.0811 [astro-ph.HE].

[11] F. Özel, D. Psaltis, T. Güver, G. Baym, C. Heinke, and S. Guillot, The Dense Matter Equation of State from Neutron Star Radius and Mass Measurements, Astrophys. J. 820, 28 (2016), arXiv:1505.05155 [astro-ph.HE].

[12] J. Nättilä, M. C. Miller, A. W. Steiner, J. J. E. Kajava, V. F. Suleimanov, and J. Poutanen, Neutron star mass and radius measurements from atmospheric model fits to X-ray burst cooling tail spectra, Astron. Astrophys. 608, A31 (2017), arXiv:1709.09120 [astro-ph.HE].

[13] T. Salmi, J. Nättilä, and J. Poutanen, Bayesian parameter constraints for neutron star masses and radii using X-ray timing observations of accretion-powered millisecond pulsars, Astron. 
Astrophys. 618, A161 (2018), arXiv:1805.01149 [astro-ph.HE].

[14] M. C. Miller, Astrophysical Constraints on Dense Matter in Neutron Stars, arXiv e-prints , arXiv:1312.0029 (2013), arXiv:1312.0029 [astro-ph.HE].

[15] M. C. Miller and F. K. Lamb, Observational constraints on neutron star masses and radii, Eur. Phys. J. A 52, 63 (2016), arXiv:1604.03894 [astro-ph.HE].

[16] T. E. Riley, A. L. Watts, S. Bogdanov, P. S. Ray, R. M. Ludlam, S. Guillot, Z. Arzoumanian, C. L. Baker, A. V. Bilous, D. Chakrabarty, K. C. Gendreau, A. K. Harding, W. C. G. Ho, J. M. Lattimer, S. M. Morsink, and T. E. Strohmayer, A NICER View of PSR J0030+0451: Millisecond Pulsar Parameter Estimation, Astrophys. J. Lett. 887, L21 (2019), arXiv:1912.05702 [astro-ph.HE].

[17] G. Raaijmakers, T. E. Riley, A. L. Watts, S. K. Greif, S. M. Morsink, K. Hebeler, A. Schwenk, T. Hinderer, S. Nissanke, S. Guillot, Z. Arzoumanian, S. Bogdanov, D. Chakrabarty, K. C. Gendreau, W. C. G. Ho, J. M. Lattimer, R. M. Ludlam, and M. T. Wolff, A Nicer View of PSR J0030+0451: Implications for the Dense Matter Equation of State, Astrophys. J. Lett. 887, L22 (2019), arXiv:1912.05703 [astro-ph.HE].

[18] A. V. Bilous, A. L. Watts, A. K. Harding, T. E. Riley, Z. Arzoumanian, S. Bogdanov, K. C. Gendreau, P. S. Ray, S. Guillot, W. C. G. Ho, and D. Chakrabarty, A NICER View of PSR J0030+0451: Evidence for a Global-scale Multipolar Magnetic Field, Astrophys. J. Lett. 887, L23 (2019), arXiv:1912.05704 [astro-ph.HE].

[19] M. C. Miller, F. K. Lamb, A. J. Dittmann, S. Bogdanov, Z. Arzoumanian, K. C. Gendreau, S. Guillot, A. K. Harding, W. C. G. Ho, J. M. Lattimer, R. M. Ludlam, S. Mahmoodifar, S. M. Morsink, P. S. Ray, T. E. Strohmayer, K. S. Wood, T. Enoto, R. Foster, T. Okajima, G. Prigozhin, and Y. Soong, PSR J0030+0451 Mass and Radius from NICER Data and Implications for the Properties of Neutron Star Matter, Astrophys. J. Lett. 887, L24 (2019), arXiv:1912.05705 [astro-ph.HE].

[20] S. De, D. Finstad, J. M. Lattimer, D. A. Brown, E. Berger, and C. M. Biwer, Tidal Deformabilities and Radii of Neutron Stars from the Observation of GW170817, Phys. Rev. Lett. 121, 091102 (2018), arXiv:1804.08583 [astro-ph.HE].

[21] B. P. Abbott et al., GW170817: Measurements of Neutron Star Radii and Equation of State, Phys. Rev. Lett. 121, 161101 (2018), arXiv:1805.11581 [gr-qc].

[22] N. Chamel and P. Haensel, Physics of Neutron Star Crusts, Living Rev. Relativity 11, 10 
(2008), arXiv:0812.3955 [astro-ph].

[23] S. Yoshida and U. Lee, Nonradial oscillations of neutron stars with a solid crust. Analysis in the relativistic Cowling approximation, Astron. Astrophys. 395, 201 (2002), arXiv:astro$\mathrm{ph} / 0210591$ [astro-ph].

[24] C. V. Flores, Z. B. Hall, and P. Jaikumar, Nonradial oscillation modes of compact stars with a crust, Phys. Rev. C 96, 065803 (2017).

[25] B. L. Schumaker and K. S. Thorne, Torsional oscillations of neutron stars, Mon. Not. R. Astron Soc. 203, 457 (1983).

[26] L. S. Finn, Non-radial pulsations of neutron stars with a crust, Mon. Not. R. Astron Soc. 245, $82(1990)$.

[27] A. J. Penner, N. Andersson, L. Samuelsson, I. Hawke, and D. I. Jones, Tidal deformations of neutron stars: The role of stratification and elasticity, Phys. Rev. D 84, 103006 (2011), arXiv:1107.0669 [astro-ph.SR].

[28] B. Biswas, R. Nandi, P. Char, and S. Bose, Role of crustal physics in the tidal deformation of a neutron star, Phys. Rev. D 100, 044056 (2019), arXiv:1905.00678 [gr-qc].

[29] S. Y. Lau, P. T. Leung, and L. M. Lin, Two-layer compact stars with crystalline quark matter: Screening effect on the tidal deformability, Phys. Rev. D 99, 023018 (2019), arXiv:1808.08107 [astro-ph.HE].

[30] N. Andersson and P. Pnigouras, The g-mode spectrum of reactive neutron star cores, Mon. Not. R. Astron Soc. 489, 4043 (2019), arXiv:1905.00010 [gr-qc].

[31] K. S. Thorne and A. Campolattaro, Non-Radial Pulsation of General-Relativistic Stellar Models. I. Analytic Analysis for $l \geq 2$, Astrophys. J. 149, 591 (1967).

[32] T. Regge and J. A. Wheeler, Stability of a Schwarzschild Singularity, Phys. Rev. 108, 1063 (1957).

[33] Y. Kojima, Equations governing the nonradial oscillations of a slowly rotating relativistic star, Phys. Rev. D 46, 4289 (1992).

[34] N. Andersson and G. L. Comer, Relativistic Fluid Dynamics: Physics for Many Different Scales, Living Rev. Relativity 10, 1 (2007), arXiv:gr-qc/0605010 [gr-qc].

[35] T. Hinderer, Tidal Love Numbers of Neutron Stars, Astrophys. J. 677, 1216 (2008), arXiv:0711.2420 [astro-ph].

[36] N. Andersson, B. Haskell, G. L. Comer, and L. Samuelsson, The dynamics of neutron star 
crusts: Lagrangian perturbation theory for a relativistic superfluid-elastic system, Class. Quantum Gravity 36, 105004 (2019), arXiv:1811.09419 [gr-qc].

[37] J. P. Pereira, M. Bejger, N. Andersson, and F. Gittins, Tidal deformations of hybrid stars with sharp phase transitions and elastic crusts, (unpublished).

[38] T. Binnington and E. Poisson, Relativistic theory of tidal Love numbers, Phys. Rev. D 80, 084018 (2009), arXiv:0906.1366 [gr-qc].

[39] T. Damour and A. Nagar, Relativistic tidal properties of neutron stars, Phys. Rev. D 80, 084035 (2009), arXiv:0906.0096 [gr-qc].

[40] K. S. Thorne, Multipole expansions of gravitational radiation, Rev. Mod. Phys. 52, 299 (1980).

[41] A. Y. Potekhin, A. F. Fantina, N. Chamel, J. M. Pearson, and S. Goriely, Analytical representations of unified equations of state for neutron-star matter, Astron. Astrophys. 560, A48 (2013), arXiv:1310.0049 [astro-ph.SR].

[42] F. Douchin and P. Haensel, A unified equation of state of dense matter and neutron star structure, Astron. Astrophys. 380, 151 (2001), arXiv:astro-ph/0111092 [astro-ph].

[43] M. Maggiore, C. den van Broeck, N. Bartolo, E. Belgacem, D. Bertacca, M. A. Bizouard, M. Branchesi, S. Clesse, S. Foffa, J. García-Bellido, S. Grimm, J. Harms, T. Hinderer, S. Matarrese, C. Palomba, M. Peloso, A. Ricciardone, and M. Sakellariadou, Science Case for the Einstein Telescope, arXiv e-prints , arXiv:1912.02622 (2019), arXiv:1912.02622 [astroph.CO].

[44] A. J. Penner, N. Andersson, D. I. Jones, L. Samuelsson, and I. Hawke, Crustal Failure during Binary Inspiral, Astrophys. J. Lett. 749, L36 (2012), arXiv:1109.5041 [astro-ph.SR].

[45] C. J. Horowitz and K. Kadau, Breaking Strain of Neutron Star Crust and Gravitational Waves, Phys. Rev. Lett. 102, 191102 (2009), arXiv:0904.1986 [astro-ph.SR].

[46] N. Andersson, G. L. Comer, and D. Langlois, Oscillations of general relativistic superfluid neutron stars, Phys. Rev. D 66, 104002 (2002), arXiv:gr-qc/0203039 [gr-qc].

[47] L.-M. Lin, N. Andersson, and G. L. Comer, Oscillations of general relativistic multifluid/multilayer compact stars, Phys. Rev. D 78, 083008 (2008), arXiv:0709.0660 [gr-qc].

[48] C. J. Krüger, W. C. G. Ho, and N. Andersson, Seismology of adolescent neutron stars: Accounting for thermal effects and crust elasticity, Phys. Rev. D 92, 063009 (2015), arXiv:1402.5656 [gr-qc]. 